\title{
XXVIII. Alternate current and potential difference analogies in the methods of measuring power
}

\section{Prof. W. E. Ayrton F.R.S. \& W. E. Sumpner D.Sc.}

To cite this article: Prof. W. E. Ayrton F.R.S. \& W. E. Sumpner D.Sc. (1891) XXVIII. Alternate current and potential difference analogies in the methods of measuring power, Philosophical Magazine Series 5, 32:195, 204-215, DOI: 10.1080/14786449108621412

To link to this article: http://dx.doi.org/10.1080/14786449108621412

Published online: 08 May 2009.

Submit your article to this journal $₫$

Џ Article views: 3

Q View related articles 5 


\section{[ 204 ]}

XXVIII. Alternate Current and Potential Difference Analogies in the Methods of Measuring Power. By Prof. W. E. Ayrton, F.R.S., and W. E. Sumpner, D.Sc.*

\section{I.}

TN a paper read by us before this Society on March 6th it 1 was pointed out that for every problem involving alternate P.Ds. in series there was an analogous problem involving alternate currents in parallel. This general proposition tells us that we can transform each of the P.D. equations given, for example, in our paper on "The Measurement of the Power given by any Electric Current to any Circuit," read before the Royal Society, April 9th, 1891, into a current equation, and so transform our method of calculating power by the measurement of three P.Us. into a method of calculating power by the measurement of three currents.

Fig. 1.

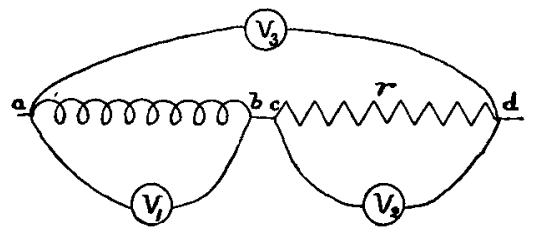

Such a transformation of our equations has been recently carried out by Dr. Fleming in the 'Electrician,' for May 8tb, and the method he arrives at as well as the three-voltmeter method of which it is an analogue are seen in figures 2 and 1 . If $\mathrm{V}_{1}, \mathrm{~V}_{3}, \mathrm{~V}_{3}$ be the readings of the voltmeters in figure 1, and $A_{1}, A_{2}, A_{3}$ the readings of the three ammeters in figure 2 , and $r$ the resistance of the non-inductive portion of the circuit $c d$ in each case, then the mean watts given to $a b$ are respectively, whatever be the nature

Fig. 2.

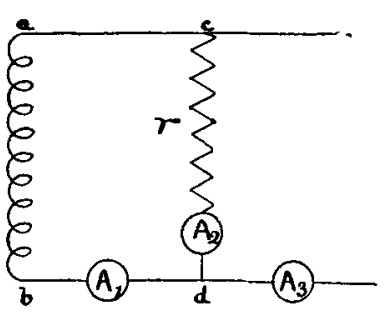

and

$$
\frac{1}{2 r}\left(\mathrm{~V}_{3}{ }^{2}-\mathrm{V}_{1}{ }^{2}-\mathrm{V}_{2}{ }^{2}\right)
$$

$$
\frac{r}{2}\left(A_{3}{ }^{2}-A_{1}{ }^{2}-A_{2}{ }^{2}\right) \text {. }
$$

* Communicated by the Physical Society: read June 12, 1891. 
The three-ammeter method has the advantage over the three-voltmeter method, in that the dynamo need not give a larger P.D. than that necessary to send the current through $a b$; it is inferior to the three-voltmeter method in that while it is possible to measure $V_{1}, V_{2}$, and $V_{3}$ rapidly in succession by using only one voltmeter, it is, of course, impossible to use only one ammeter to measure $A_{1}, A_{2}$, and $A_{3}$ without constantly interrupting the circuit, and hence it would be necessary to accurately calibrate three instruments if this current method were employed.

But the main objection to this current method is that, as Dr. Fleming points out, it does not possess the accuracy of our three-voltmeter method of measuring power. For in order that this three-current method may give accurate results it is necessary to assume, to quote from our Royal Society paper, "the entire absence of self and mutual induction from a circuit some portion of which is necessarily of a solenoidal form."

It is possible, however, to obtain a current analogue of our three-voltmeter method which shall have the accuracy of the three-voltmeter method itself. And as the general proposition given in our previous paper tells us that the current analogues of P.D. arrangements in series are current arrangements in parallel, it follow's that with this other method also the dynamo will not be required to produce a greater P.D. than that necessary to send the current through the circuit the power given to which we desire to measure.

The method is as follows:-In parallel with the circuit $a b$ (fig. 3) the power given to which we wish to measure connect a non-inductive resistance of $r$ ohms (in circuit with which no instrument is placed, which would necessarily make the so-called. non-inductive branch more or less inductive). Let $A_{3}, A_{1}$, and $\mathrm{V}$ be the readings of the two ammeters and the voltmeter placed

Fig. 3.

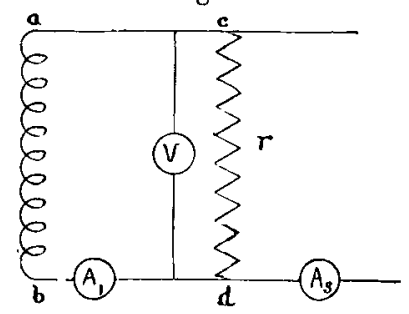
as shown, then, from the equations given in our Royal Society paper, it follows at once that the mean watts given to $a b$ are

$$
\frac{r}{2}\left\{\mathrm{~A}_{3}{ }^{2}-\mathrm{A}_{1}{ }^{2}-\left(\frac{\mathrm{V}}{r}\right)^{2}\right\} \text {. }
$$

It is interesting to notice that if $a b$ were the primary coil of a transformer, it would be when the load on the secondary 
was small, that is when the current passing through $a b$ was small, that it would be most difficult, on account of lag, to measure with ordinary methods the power given to $a b$. But that is exactly the case when it is most easy to use our one-voltmeter and two-ammeter method, since when the dynamo has to send little current through $a b$ there is little objection to requiring it to send a current through $c d$ in parallel with $a b$.

If the voltmeter (fig. 3) be a hot wire instrument, then, since an appreciable current will pass through this voltmeter, $r$ must be taken as the parallel resistance of $c d$ and of the voltmeter. It is important to observe, however, that there is no necessity to know either of these resistances separately, since the value of $r$ can be determined when the three instruments $A_{1}, A_{3}$, and $V$ are relatively calibrated thus :-First, break the circuits of $c d$ and of the voltmeter, and compare the deflexion of $A_{3}$ with $A_{1}$; this calibrates ammeter $A_{3}$ relatively to ammeter $A_{1}$, the calibration of which we will assume to be correct; second, close the circuits of $c d$ and of the voltmeter, but break the transformer circuit $a b, A_{3}$ is now in series with the parallel circuit containing $c d$ and the voltmeter. The value of $r$ is therefore at once known, since $r$ must equal the volts as read by $\mathrm{V}$ divided by the amperes as read by $A_{3}$.

\section{II.}

As an illustration of the general proposition to which we have referred, it may interest the Members to see what are the other analogies that we have traced out between alternate P.Ds. in series and alternate currents in parallel in connexion with the measurement of power.

Fig. 4.

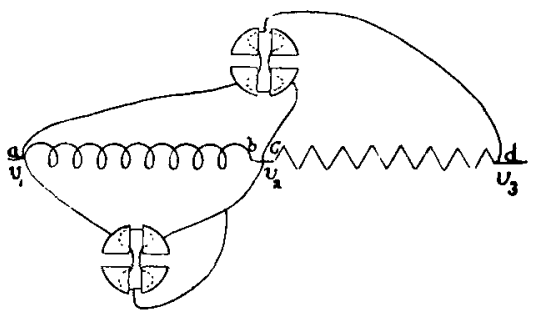

Mr. Blakesley's method, communicated to this Society in February of this year * , is the current analogue of our electrometer method of 1881. For with the electrometer method (fig. 4) we make two measurements, one giving us the mean

* Phil Mag. April 1891, p. 346. 
value of

the other the mean value of

$$
\left(v_{1}-v_{2}\right)\left(v_{3}-\frac{v_{1}+v_{2}}{2}\right)
$$

$$
\frac{\left(v_{1}-v_{2}\right)^{2}}{2}
$$

$v_{1}, v_{2}, v_{3}$ being the instantaneous values of the potentials. Then we take the difference, and so get the mean value of

$$
\left(v_{1}-v_{2}\right)\left(v_{2}-v_{3}\right) \text {, }
$$

which is equal to $r$ times the mean watts given to $a b$ (fig. 4).

With Mr. Blakesley's method two measurements are made, one with a split dynamometer (fig. 5) giving the mean value of

$$
\alpha_{1} \alpha_{3}
$$

Fig. 5.

and the other with an ammeter giving the mean value of

$$
\alpha_{1}^{2}
$$

$\alpha_{1}, \alpha_{2}, \alpha_{3}$ being the instantaneous values of the currents. Then the difference is taken, and so the mean value of

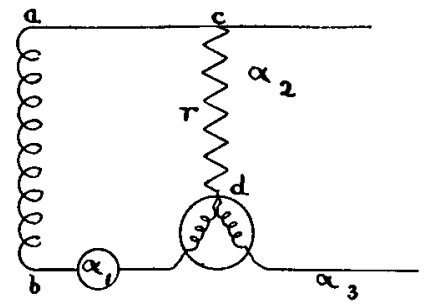

$$
\alpha_{1}\left(\alpha_{3}-\alpha_{1}\right) \text { or } \alpha_{1} \alpha_{2}
$$

is obtained, and this is equal to $\frac{1}{r}$ times the mean watts given to $a b$ (fig. 5 ).

It is important to notice that as no instrument is inserted in the non-inductive circuit $a b$ (fig. 5), this method of Mr. Blakesley's has exactly the same accuracy as the electrometer method.

III.

The electrometer measurements may, as we pointed out some years ago, be varied; and by making the connexions as seen in fig. 6 , we can obtain from the two readings the mean values of

and

$$
\left(v_{2}-v_{3}\right)\left(v_{1}-\frac{v_{2}+v_{3}}{2}\right)
$$

$$
\frac{\left(v_{2}-v_{3}\right)^{2}}{2}
$$


then, taking the difference, we get the mean value of

$$
\left(v_{2}-v_{3}\right)\left(v_{1}-v_{2}\right)
$$

which, as before, is equal to $r$ times the mean watts given to $a b$.

Fig. 6.

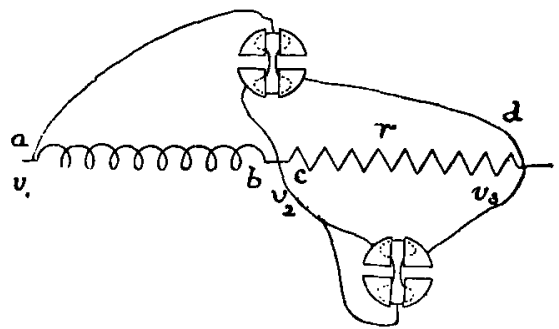

Mr. Blakesley has also pointed out, in his paper published in the Phil. Mag. for April of this year, that the current measurements may be varied and the apparatus arranged as seen in fig. 7. The two measurements now give respectively the mean values of

and

$$
\alpha_{2} \alpha_{3}
$$

$$
\alpha_{2}{ }^{2} \text {; }
$$

therefore the difference gives the mean value of

$$
\alpha_{2}\left(\alpha_{3}-\alpha_{2}\right) \text {, }
$$

Fig. 7.

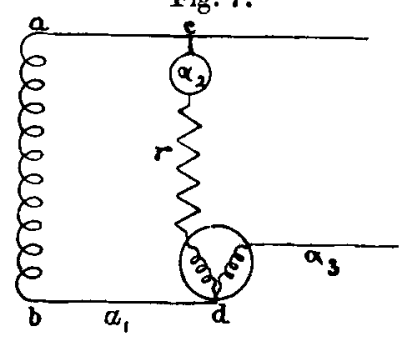

that is, the mean value of

$$
\alpha_{2} \alpha_{1}
$$

which is equal to $\frac{1}{r}$ times the mean watts given to $a b$.

While, however, our second method of using the electrometer (fig. 6) gives the answer with the same accuracy as the first method (fig. 4), Mr. Blakesley's second method of joining up the dynamometers in figure 7 introduces selfinduction into a circuit which ought to be entirely noninductive, and so it does not give the answer with the same accuracy as his first method (fig. 5).

\section{IV.}

The modification of our electrometer method suggested to one of us by Mr. L. Atkinson, while he was a pupil at the Finsbury Technical College, and afterwards carried out by 
MM. Blondlot and Curie, of making an electrometer with two needles for the measurement of power, is the exact analogue of the wattmeter method. For with the doubleneedle electrometer (fig. 8) we obtain from a single reading

Fig. 8.

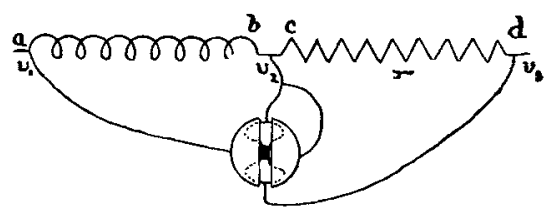

the mean value of

$$
\left(v_{1}-v_{2}\right)\left(v_{2}-v_{3}\right) \text {, }
$$

which is equal to $r$ into the mean watts given to $a$. Similarly with the wattmeter (fig. 9) we obtain from a single reading the mean value of

Fig. 9.

$$
\alpha_{1} \alpha_{3},
$$

which is proportional to $\frac{1}{r}$ into the mean watts given to $a b$.

While, however, the double-needle electrometer gives us the answer with perfect accuracy, the wattmeter method is liable to inaccuracy from the circuit $e d$ not being strictly non-inductive.

Some years ago, at a meeting of the Institution of Electrical Engineers, one of us published the formula for the error made in using a wattmeter to measure the power given by an alternate current to an inductive circuit. Not wishing to cumber the pages of scientific periodicals with elementary mathematics, it was thought sufficient merely to state this formula without publishing a proof. But as our formula has now been introduced into text-books, and as the appropriation thereof by the writer of a well-known treatise has led him to supply a proof of it involving an appalling array of mathematical equations, we venture to offer a proof which, although very simple, is perfectly accurate. We are the more induced to do this because we find that this formula, and its proof for the error due to self-induction in the supposed non-inductive portion of the circuit $c d$, apply equally well to all the nine methods of measuring power illustrated in figures 1 to 9 of this paper.

Plit. Mag, S. 5. Vol. 32. No. 195. August 1891. P 
The formula employed with such methods for giving the mean watts, whether it involves the reading of one instrument, as in the case of the wattmeter (fig. 9), or of two instruments, as with the methods illustrated in figures $4,5,6,7$, and 8 , or of three instruments, as with the methods illustrated in figures 1, 2, and 3, gives with perfect accuracy $r$ times the mean product of two currents, or $\frac{1}{r}$ times the mean product of two P.D.s. Whether this mean product is directly proportional to the mean watts given to $a b$ depends in all the nine cases on the following consideration :-

The mean product between two currents which are sine functions of the time is, as every student now knows, equal to half the product of their maximum values into the cosine of the phase angle between them. Therefore if the angle of lag between the current in $a b$ and the P.D. between its terminals be $\theta$, and the angle of lag between the current in $c d$ and the P.D. between its terminals be $\phi$, and if the maximum values of the currents in these two circuits be $\mathrm{A}_{1}{ }^{\prime}$ and $\mathrm{A}_{2}{ }^{\prime}$ respectively, and the maximum values of the P.D.s at the terminals of these circuits be $V_{1}^{\prime}$ and $V_{2}^{\prime}$, it follows that the formula used to measure the watts in the cases $2,3,5,7$, and 9 gives

$$
r \frac{\mathrm{A}_{1}{ }^{\prime} \mathrm{A}_{2}{ }^{\prime} \cos (\theta-\phi)}{2},
$$

and in the cases $1,4,6$, and 8 ,

$$
\frac{\nabla_{1}^{\prime} V_{2}^{\prime} \cos (\theta-\phi)}{2 r}
$$

But what we want to measure is the mean product of the current in al, into the P.D. between its terminals, and this product equals

$$
\frac{A_{1}^{\prime} V_{1}^{\prime} \cos \theta}{2}
$$

But

$$
{ }^{r} \mathrm{~A}_{2}^{\prime}=\mathrm{V}_{2}^{\prime} \cos \phi
$$

and in the methods illustrated in the figures $2,3,5,7$, and 9

$$
\mathrm{V}_{2}{ }^{\prime}=\mathrm{V}_{1}^{\prime} \text {, }
$$

while in the methods illustrated in the figures $1,4,6$, and 8

$$
A_{2}{ }^{\prime}=A_{1}{ }^{\prime} \text {; }
$$


thercfore in all the nine cases

$$
\begin{aligned}
\frac{\text { Apparent watts }}{\text { True watts }} & =\frac{\cos (\theta-\phi) \cdot \cos \phi}{\cos \theta} \\
& =\frac{1+\tan \theta \cdot \tan \phi}{1+\tan ^{2} \phi} . . .
\end{aligned}
$$

The circuit $c d$ need only possess a self-induction $l$, even if an ammeter or dynamometer form part of it, but the circuit $a b$ may have mutual induction and capacity as well as selfinduction. If therefore we write expression (1) in the form

$$
\frac{1+\frac{\mathrm{L} p}{\mathrm{R}} \cdot \frac{l p}{r}}{1+\left(\frac{l p}{r}\right)^{2}}, \cdot \text {. . . . . . }
$$

as we did in 1888 , where $p$ equals $2 \pi$ times the frequency, it must be remembered that while $l$ and $r$ are the true values of the self-induction and resistance of $c d, \mathrm{~L}$ and $\mathrm{R}$ are only the effective self-induction and resistance of $a b$. Hence, as Mr. Blathy suggested in the 'Electrician' for 1888, it is better to leave our expression for the ratio of the apparent to the true watts in the general form as given in (1) rather than to put it in the derived form as given in (2).

$\phi$ will generally be positive if the resistance of $c d$ is small ; but, if $e d$ contains a doubly-wound high resistance-coil, as is generally the case when $c d$ is the fine-wire circuit of a wattmeter, then it is quite possible to make $\phi$ positive, nought, or negative. $\theta$ may, of course, be also positive, nought, or negative, depending on whether the self and mutual induction effects preponderate or not over the capacity effect. It is therefore possible to have either $\theta$ or $\phi$, or both, positive or negative.

The apparent watts will therefore be :-

too large if $\theta$ and $\phi$ be both of the same sign and $\theta>\phi$;

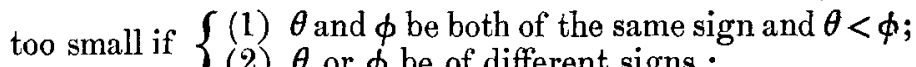

$\{$ (2) $\theta$ or $\phi$ be of different signs ;

correct if $\{(1) \theta$ and $\phi$ be equal;

$\{$ (2) $\phi$ be nought.

Now $\phi$ can be made very small in one or other of three ways :-

1. Use some method of testing, like that shown in figures $1,3,4,5,6$, and 8 , which does not require any measuring instrument to be placed in the noninductive circuit $e d$. 
2. Use a wattmeter (fig. 9), and make the capacity of the stationary doubly-wound resistance-coil exactly balance the self-induction of the suspended and the stationary coils.

3. Make the resistance of the fine-wire circuit, $c d$, of the wattmeter small. For with a given P.D. between the terminals of $c d$ the same deflexion of the measuring instrument can be obtained for different values of the resistance of $c d$ if we make the number of turns in the coil or coils of the measuring instrument in $c d$ proportional to the resistance $c d$. But the selfinduction of the coil or coils is proportional to the square of the number of turns, and therefore proportional to the square of $r$ for a given deflexion of the measuring instrument. Hence $\tan \phi$ can be made as small as we like for a given value of $p$ by making the resistance of $c d$ small.

This suggests a current method of measuring the power given to any circuit which is no more wasteful of power than the methods shown in figures $2,3,5$, and 7 ; and which, although not so accurate as those shown in figures 3 and 5 , is as accurate as those shown in figures 2 and 7 . The method is simply to use a wattmeter (fig. 9), but having both its coils made of thick wire, or, as this may be called a split dynamometer, the method consists in using a split dynamometer having one of its coils in the circuit $a b$ (the power given to which we desire to measure) and the other coil in a circuit $a d$ parallel to $a b$. The power will be given at once by $r$ times the reading of the instrument and with but a very small error if $r$ be small.

\section{$\nabla$.}

Mr. Rimington has suggested a method of measuring the mean value of the product $\left(v_{1}-v_{2}\right)\left(v_{2}-v_{3}\right)$ (fig. 9) by means of a dynamometer, each of whose coils is in circuit with a high resistance, joined up as shown in figure 10.

Fig. 10.

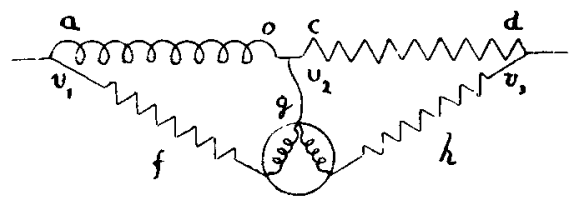

The objection to this method is as follows:-By making the time-constants of each of the circuits of the dynamometer a $f g$, 
$d h g$ equal to one another, we can, no doubt, make the difference of phase in the two currents passing through the dynamometer exactly the same as the difference in phase between the current through $a b$ and the P.D. at the terminals of $a b$; but we cannot make the currents through the dynamometer coils independent of the rate of alternation. Hence, if this instrument be employed for measuring the power given to $a b$ in the way shown in figure 10 , it must be calibrated for each rate of alternation of the current.

But although this defect exists in the employment of Mr. Rimington's high-resistance split dynamometer for the measurement of power, it can be used without error for measuring the phase-angle between two P.D.s in series by a method analogous to that employed by Mr. Blakesley for measuring the phase-difference between two currents in parallel.

Fig. 11 shows Mr. Blakesley's method: the dynamo-

Fig. 11.

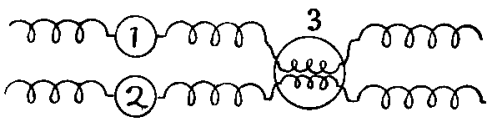

meters 1 and 2 give respectively the mean squares of the currents in the two circuits, and the dynamometer 3 the mean product of the two currents; and from the three readings we have, as is now well known,

$$
\cos \theta=\sqrt{\frac{\text { Square of reading of } 3}{\text { Reading of } 1 \times \text { Reading of } 2}},
$$

where $\theta$ is the angle of lag.

Fig. 12.

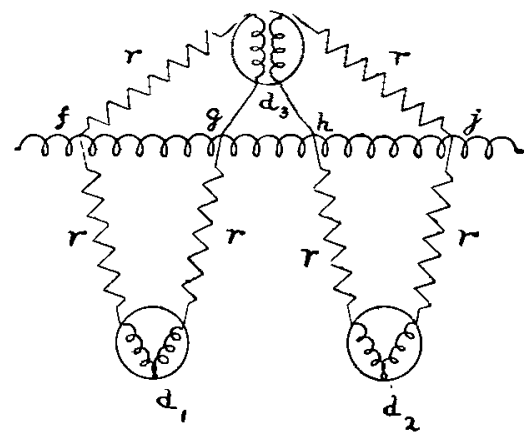

Now let $f g$ and $h j$ be two circuits in series (fig. 12), and let it be required to find the angle of phase-difference $\theta$ 
214 Alteriate Current and Potential Difference Analogies.

between the P.D. at the terminals of $f g$ and the P.D. at the terminals of $h j$. Connect up the high-resistance dynamometer successively as shown, and let $d_{1}, d_{2}$, and $d_{3}$ be the three deflexions obtained.

Each circuit of the dynamometer consists of a coil of fine wire, and a non-inductive high resistance in series with it. Let $*$ be the total resistance of each circuit, and let $\phi$ be the angle of lag between a current in either cireuit of the dynanometer and the P.D. at its terminals.

Let $V_{1}^{\prime}$ and $V_{2}^{\prime}$ be the maximum values of the P.D. between $f$ and $g$, and between $h$ and $j$ respectively, then

$$
\begin{aligned}
d_{1} \propto \frac{1}{2}\left(\frac{V_{1}^{\prime}}{2 r}\right)^{2} \cos ^{2} \phi, \\
d_{2} \propto \frac{1}{2}\left(\frac{V_{2}^{\prime}}{2 r}\right)^{2} \cos ^{2} \phi, \\
d_{3} \propto \frac{1}{2}\left(\frac{V_{1}^{\prime}}{r} \cos \phi \times \frac{V_{2}^{\prime}}{r} \cos \phi\right) \cos \theta, \\
\therefore \quad \cos \theta=\sqrt{\frac{d_{3}{ }^{*}}{16 d_{1} d_{2}} .}
\end{aligned}
$$

Figure 13 shows the way in which the Blondlot and Curie double-needle electrometer can be successively connected up

Fig. 13.

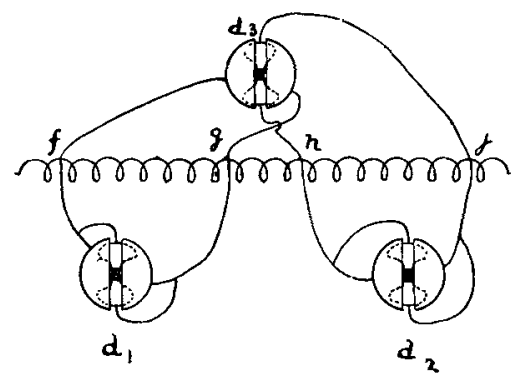

so as to obtain, from the readings $d_{1}, d_{2}, d_{3}$, the angle of phase-difference between the P.D. at the terminals of $f g$, and the P.D. at the terminals of $h j$, the formula being, of course,

$$
\cos \theta=\sqrt{\frac{d_{3}^{2}}{d_{1} \times d_{2}}}
$$

I we desire to measure the angle of lag between the current in any circuit $a b$ and the P.D. between its terminals, we 
Mr. W. Sutherland on a Kinetic Theory of Solids. $\quad 215$

can employ either the three-voltmeter method (fig. 1) or either of its unalogues, viz. the three-ammeter method (fig. 2), or the one-voltmeter and two-ammeter methor (fig. 3). The formula giving the cosine of the lag angle for the three methods are, respectively,

$$
\begin{aligned}
& \cos \theta=\frac{\mathrm{V}_{3}{ }^{2}-\mathrm{V}_{1}{ }^{2}-\mathrm{V}_{2}{ }^{2}}{2 \mathrm{~V}_{1} \mathrm{~V}_{2}}, \\
& \cos \theta=\frac{\mathrm{A}_{3}{ }^{2}-\mathrm{A}_{1}{ }^{2}-A_{2}{ }^{2}}{2 \mathrm{~A}_{1} \mathrm{~A}_{2}}, \\
& \cos \theta=\frac{A_{3}{ }^{2}-A_{1}{ }^{2}-\left(\frac{V}{r}\right)^{2}}{2 \mathrm{~A}_{1} \frac{V}{r}},
\end{aligned}
$$

$V_{1}, V_{2}, V_{3}, A_{1}, A_{2}, A_{3}$, and $V$ being the readings of the instruments in the different cases.

\section{A Kinetic Theory of Solids, with an Experinental Introduction. By William SuthenLaxd.}

$$
\begin{aligned}
& \text { [Continued from p. 43.] } \\
& \text { Young's Modulus. }
\end{aligned}
$$

1. The experimental determinations that have hitherto been made of the temperature variations of Young's Modulus have been made for the most part by the static method, the difficulties of which detract from its trustworthiness; for example, Wertheim, by the static method, found the Young's Modulus of iron and silver to increase with rising temperature, though we now know that it diminishes. Kupffer (Mém. de l'Acad. Impér. des Sc. de St. Pét. 6 sér. t. 6) devised a convenient modification of the kinetic method (by lateral vibrations) which lent itself well to the measurement of the temperature effect, but used an erroneous formula for calculating the modulus from his measurements. Zöppritz (Pogg. Ann. exxviii.) has recalculated with a correct theory the right values of the modulus of various metals at ordinary temperature from Kupffer's data. I recalculated the values at other temperatures, but found the temperature interval of about $30^{\circ} \mathrm{C}$. too small to give trustworthy results. Accordingly I undertook a series of measurements by Kupffer's method.

The method consists in causing a rod of the metal to vibrate laterally with one end clamped and the other free, first with the free end vertically above the clamped one, and next under it. 